Journal of Engineering and Applied Sciences 14 (Special Issue 9): 10740-10757, 2019

ISSN: 1816-949X

(C) Medwell Journals, 2019

\title{
MC-DS-CDMA System Based on DWT and STBC in Multipath Fading ITU Channels Model
}

\author{
${ }^{1,2}$ Nader Abdullah Khadam, ${ }^{1}$ Laith Ali Abdul-Rahaim, ${ }^{2}$ Arash Ahmadi, \\ ${ }^{2}$ Seyed Vahab Makki and ${ }^{2}$ Golamreza Karimi \\ ${ }^{1}$ Department of Electrical Engineering, College of Engineering, \\ University of Babylon, Hillah, Iraq \\ ${ }^{2}$ Department of Electrical Engineering, College of Engineering, \\ Razi University, Kermanshah, Iran
}

\begin{abstract}
In this study, the performance of MultiCarrier Direct Sequence Code Division Multiple Access (MC-DS-CDMA) in fixed MC-DS-CDMA and mobile MC-DS-CDMA applications have been improved by using the compensations of space time block coding and Discrete Fast Fourier Transform (FFT) or Discrete Wavelets Transform DWT. These MC-DS-CDMA systems had been simulated using MATLAB 2015 a. Through simulation of the proposed system, various parameters can be changed and tested. The Bit Error Rate (BERs) of these systems are obtained over wide range of signal to noise ratio. All simulation results had been compared with each other using different subcarrier size of FFT or DWT with STBC for 1, 2, 3 and 4 antennas in transmitter and under different ITU multipath fading channels and different Doppler frequencies (fd). The proposed structures of STBC-MC-DS-CDMA system based on DWT batter than based on (FFT) in varies Doppler frequencies and subcarrier size. Also, proposed system with STBC based on 4 transmitters better than other systems based on 1 or 2 or 3 transmitters in all Doppler frequencies and subcarrier size in all simulation results. Finally, digital hardware designed for transmitter and receiver of STBC-MC-DS-CDMA algorithm to prove the efficiency of the system techniques using FPGA type to check and optimized the chip area of implementation of proposed system.
\end{abstract}

Key words: MC-DS-CDMA, STBC, ITU channels model, FFT, DWT, OFDM

\section{INTRODUCTION}

Wireless communication is considered as one of the big engineering success stories over the last two decades. It is one of the rapidly changing fields that demand for faster data rates with longer transmission ranges to meet the standards of new applications (Linfoot et al., 2007). Wireless technologies such as cellular systems and wireless LANs, along with devices such as small portable computers, PDAs and mobile phones have enabled the growth of wireless networking such that now mobile users expect to be able to use the same multimedia applications as fixed users. Satellite networks can provide global coverage for urban and remote areas with low traffic density. Cellular networks such as Universal Mobile Telecommunications Systems (UMTS) (Shadmand et al., 2011) can cover macro cells of rural or suburban areas with low or medium traffic density. An alternative for macro coverage is Wireless Metropolitan Area Networks (WMANs) such as IEEE 802.16 (Kumbasar and Kucur, 2012). Multicarrier Direct Sequence Code Division Multiple Access (MC-DS-CDMA) is one of the newest technologies in broadband wireless access which enables high speed fixed wireless communication. Broadband wireless access provides high-rate wireless communications between a fixed access point and multiple terminals (Yan and Liu, 2004). MC-DS-CDMA is defined the standard of point-to-multipoint wireless networking. MC-DS-CDMA has the capability that connects to the ISP (Internet Service Provider) even when you are roaming outside home or office. The MC-DS-CDMA technology is becoming the way to avert the impending crisis of rural connectivity (Izumi et al., 2007). MC-DS-CDMA is intended for wireless Metropolitan Area Networks (MAN). In a MAN, the MC-DS-CDMA can provide Broadband Wireless Access (BWA) up to 30 miles $(50 \mathrm{~km})$ for fixed stations and 3-10 miles (5-15 km) for mobile stations (Chen and Tsao, 2012). STBC Based MC-DSCDMA based solutions are more flexible and secured. STBC based MC-DS-CDMA technology is 30 times faster than the third generation and 100 times faster than the wireless data rates. The two-main application of STBC based MC-DS-CDMA are fixed STBC based MC-DS-CDMA which are point to multipoint enabling broadband access to homes and offices and mobile STBC 


\section{J. Eng. Applied Sci., 14 (Special Issue 9): 10740-10757, 2019}

based MC-DS-CDMA which offers the full mobility of cellular networks at true speeds. STBC based MC-DS-CDMA provide fixed, nomadic, portable and mobile wireless broadband connectivity without the need for a direct line-of-sight (Shi et al., 2009). One way to effectively combat the multipath channel impairments and still provide high-data rates in a limited bandwidth is the use of an Orthogonal Frequency-Division Multiplexing (OFDM) modulation method and multiple antennas at the transmitting end (Shi and Yang, 2014; Daoud, 2012). In short, OFDM delivers a wireless signal much farther with less interference than competing technologies (Abdul-Rahaim, 2015a, b). Furthermore, the Inter-Symbol Interference (ISI) and Inter Carrier Interference (ICI) can be easily eliminated by inserting a Cyclic Prefix (CP) in front of each transmitted OFDM block (Ali and Abdul-Rahaim, 2008). Transmitter diversity can effectively combat multipath channel impairments due to the dispersive wireless channel that can cause deep fades in some sub channels (Dawood et al., 2015a, b). The combination of the two techniques, OFDM and transmitter diversity can further enhance the data rates in a frequency-selective fading environment. One attractive approach to transmit diversity is space time block coding (Chisab and Shukla, 2014; Simsir and Taspinar, 2017) in which full diversity is achieved while a very simple maximum likelihood decoding algorithm is used at the decoder. An STBC is usually represented by a matrix each row represents a time slot and each column represents one antenna's transmissions over time. It was shown by Simsir and Taspinar (2017) that there exists no complex orthogonal code which satisfies the full diversity and the full transmit rate simultaneously in more than 3 transmit antenna. In other words, not only can the two-antenna transmission system to get the full diversity and full transmission rate and this symbol as planned Alamouti scheme (Chisab and Shukla, 2014). Therefore, systems employing 3 or more transmit antennas need to sacrifice either diversity or transmission rate. The Wavelet Transforms (WT) is inserted in OFDM transmitter in order to introduce many versions for central carrier frequency providing some sort of frequency diversity that is capable of mitigating time variant fading channel. The Wavelet Transforms (WT) representation basically involves the decomposition of the signals in terms of small wave components called wavelets. It has the ability to compact the signal energy into few large coefficients. The original signal can be reconstructed perfectly from these few coefficients while suppressing the other coefficients without losing most of the features of the signal (Shi and Yang, 2014; Nagaradjane et al., 2013; Zakaria and Ruyet, 2014). There are many previous works duels with the design and evaluation of potential gains of LTE or OFDM system. Linfoot et al. (2007) propose a novel approach to transmit parallel information through a terrestrial channel that could render it a more flexible alternative to OFDM in the future using orthogonal wavelet division multiplex but there work still have many limitations in BER performance and their step of design. Shadmand et al. (2011) propose the use of wavelet transform in LTE cellular systems with mathematical expressions are derived to represent data rate in LTE downlink transmission based on wavelet and fourier transforms and the get good results for small range of multicarrier. By Shadmand et al. (2011), the performance of Wavelet based OFDM (WOFDM) is studied and compared to that of conventional OFDM over multipath Rayleigh fading channels with exponential power delay profile. By Yan and Liu (2004), proposed a new method to design OFDM based on multiwavelet packet. The design has more much lower side lobes and flexible data rate and more band efficiency and lower peak-to-average power ratio. By Daoud (2012), provides a way to improve the MIMO of OFDM system by proposing a new way to address the reduction of the Proportion of peak-to-Average Power (PAPR). This reference adds only the using of MIMO technique in OFDM based wavelet transform. By Abdul-Rahaim (2015a, b), presents a new proposed structure for WIMAX based on Discrete Wavelets Transform (DWT) as multicarrier and space time block coding. The new proposed structures improve the performance of Bit Error Rate (BER) in many channel models. Dawood et al. (2015a, b) show Discrete Multi Wavelet Critical Sampling Transform (DMWCST) has been proposed instead of Fast Fourier Transform (FFT) in the realization of the Orthogonal Frequency Division Multiplexing (OFDM) system. Dawood et al. (2015) show turbo codes effectiveness as new approach for an OFDM system based on "Discrete Multiwavelet Critical-Sampling Transform OFDM-DMWCST" and how useful of using this technique to providing good BER performance at higher data rates. A proposed novel method based on the Multiwavelet Transform MWT for applying the OFDMA in LTE have been given by Chisab and Shukla (2014). Bagadi et al. (2016) presents new technique to improve BER performance using "space time block coding multicarrier direct sequence world interoperability for microwave access STBC-MC-DS-WIMAX" based on "Discrete Wavelet Transform DWT and Phase Matrix (PM)" at high data rate. This study given two models for phase matrix and did not using 2D transformations in phase matrix model. Abdul-Rahim (2015a, b) presents new proposed structures for LTE based on "Space Time Block Coding (STBC-LTE) and Discrete Wavelets Transform (DWT) as multicarrier". The purpose of these new proposed structures is to improve the performance of Bit Error Rate (BER) compared with the conventional LTE. Ahmadinejad and Talebi (2016) proposes DMWT based orthogonal modulator. By DMWT implementing, 


\section{J. Eng. Applied Sci., 14 (Special Issue 9): 10740-10757, 2019}

a good spectral efficiency and BER can be achieved compare to FFT and DWT. Lavanya and Madheswaran (2014) proposed a work to achieve good spectral efficiency and reduction of interference by introducing multiwavelet with MIMO technology to reduction. BER for a given SNR. And also given Implementation of the proposed scheme in FPGA.

\section{MATERIALS AND METHODS}

The proposed STBC based MC-DS-CDMA systems: The STBC based MC-DS-CDMA can implement using Discrete Fourier Transform (DFT) or Fast Fourier Transforms (FFT) as complex exponential functions. So, it can be replaced by Discrete Wavelets Transform (DWT). This replacement will decrease the interference level. From many research, it's found that uses of discrete orthonormal wavelets will reduce the ICI and ISI because the DWT will Strengthens the orthogonality between the subcarrier (Abdul-Rahaim, 2015a, b). The simulation results by Ali and Abdul-Rahaim (2008), Dawood et al. (2015a, b) show this idea of replacement FFT by DWT in some multicarrier system and calculate the BER performance with these orthogonal bases. The simulations of STBC based MC-DS-CDMA system with new transform have shown the dependence of channel on the performance of DWT and FFT. The main idea for using DWT in STBC based MC-DS-CDMA system is the excellent spectral containment wavelet filters properties over Fourier filters. Under certain channel conditions, it has been found that DWT based MC-DS-CDMA does outperform better than FFT based MC-DS-CDMA. The implementations of MC-DS-CDMA by using FFT and its inverse operation IFFT in practice today (or DWT and IDWT) to substitute multicarrier operations. The Inter Symbol Interference (ISI) can be eliminated almost completely by adding a guard time interval in each packet of MC-DS-CDMA frame and this will cause a lose about $25 \%-40 \%$ from data rate and this is one of the disadvantage of FFT-MC-DS-CDMA. So, the uses of DWT instead of DFT will increase the orthogonality between the subcarrier of the MC-DS-CDMA packet and will be combat the narrowband interference and so, no need to adding a guard time interval (Chisab and Shukla, 2014; Simsir and Taspinar, 2017). The second proposed idea to MC-DS-CDMA system is adding Space Time Blocks Coding (STBC) to the system. The STBC reduce the effect of multipath frequency selective multipath fading channel. The aims this study are designing a wireless communication system with least Bit Error Rate (BER) for high data rate to fix stationary nodes and mobile users under multichannel models. These ideas will be implemented in MC-DS-CDMA system by adding STBC with more than two antennas and using DFT or DWT (Linfoot et al., 2007; Bagadi et al.,
2016; Nagaradjane et al., 2013). The proposed STBC-MC-DS-CDMA transceiver is shown in Fig. 1. All the type of space-time block codes with three transmitters or more has a coding rate of $1 / 2$ to satisfy orthogonality condition. The space-time block code for four transmits antennas $\mathrm{N}=4$ with input Symbols $\left(\mathrm{S}_{1}, \mathrm{~S}_{2}, \mathrm{~S}_{3}, \mathrm{~S}_{4}\right)$, the output will be over $\mathrm{T}=8$ symbol periods, thus, the coding Rate $\mathrm{R}=1 / 2$ (Zakaria and Ruyet, 2014; Zarrinkoubm, 2014). At a given symbol period, four antennas transmitted four signals simultaneously. At time slot $T_{0}$, transmitted signal from first Transmitter $\left(T_{x 1}\right)$ is denoted by $S_{1}$ the signal from second Transmitter $\left(T_{x 2}\right)$ by $\mathrm{S}_{2}$ and the signal from third Transmitter $\left(\mathrm{Tx}_{3}\right)$ by $\mathrm{S}_{3}$ and the signal from fourth Transmitter $\left(\mathrm{T}_{\mathrm{x} 4}\right)$ by $\mathrm{S}_{4}$. This process will go on in the same manner for each time slot until transmitting the last row of Table 1 . This table has a rate of (1/2) and encode any complex signal constellations to transmit antennas (Peng et al., 2013; Anonymous, 2011). For the four transmit and one receive antenna system, the channel coefficients are modeled by complex multiplicative distortions, $\mathrm{h}_{1}$ for the first transmit antenna $h_{2}$ for the second transmit antenna and $h_{3}$ for the third transmit antenna and $\mathrm{h}_{4}$ for the fourth transmit antenna (Abdul-Rahaim, 2015a, b). Since, some models used in this work are time varying and frequency selective for wide band mobile communication systems, so, a dynamic estimation of channel is necessary to compensate STBC based MC-DS-CDMA signal (Yan and Liu, 2004). The DWT computation in the implementation of STBC based MC-DS-CDMA systems satisfy the multiresolution conditions. In this case, "the lower resolution coefficients can be calculated from the higher resolution coefficients by a tree-structured algorithm called filter-bank” (Dawood et al., 2015a, b). The multiresolution idea is better understood as shown by Dawood et al. (2015a, b), Chisab and Shukla (2014). The computation steps for a single level DWT and a single level IDWT for 1-D signal was shown by Ahmadinejad and Talebi (2016).

The channel transfer function estimation and the inverse of it are applied to each STBC based MC-DSCDMA packet to reduce the channel effects, much like equalization (Dawood et al., 2015a, b). There are two types of channel estimations, block type and comb-type pilot channel estimation as shown by Simsir and Taspinar (2017). After pilot-carrier (training sequence) is generated as a bipolar sequence $\{ \pm 1\}$, the receiver previously knows this sequence. So, the system can estimate the channel transfer function $\mathrm{h}_{1}(\mathrm{t}), \mathrm{h}_{2}(\mathrm{t}), \mathrm{h}_{3}(\mathrm{t})$ and $\mathrm{h}_{4}(\mathrm{t})$. The inverse of these channels can be calculated. Using channels and there inverse to compensate the received packet and reduce the errors. The training sequence will be inserted in each STBC stream of the transmitter. Training sequences will be added in the receiver to form single training sequence, then spread the training sequences and 


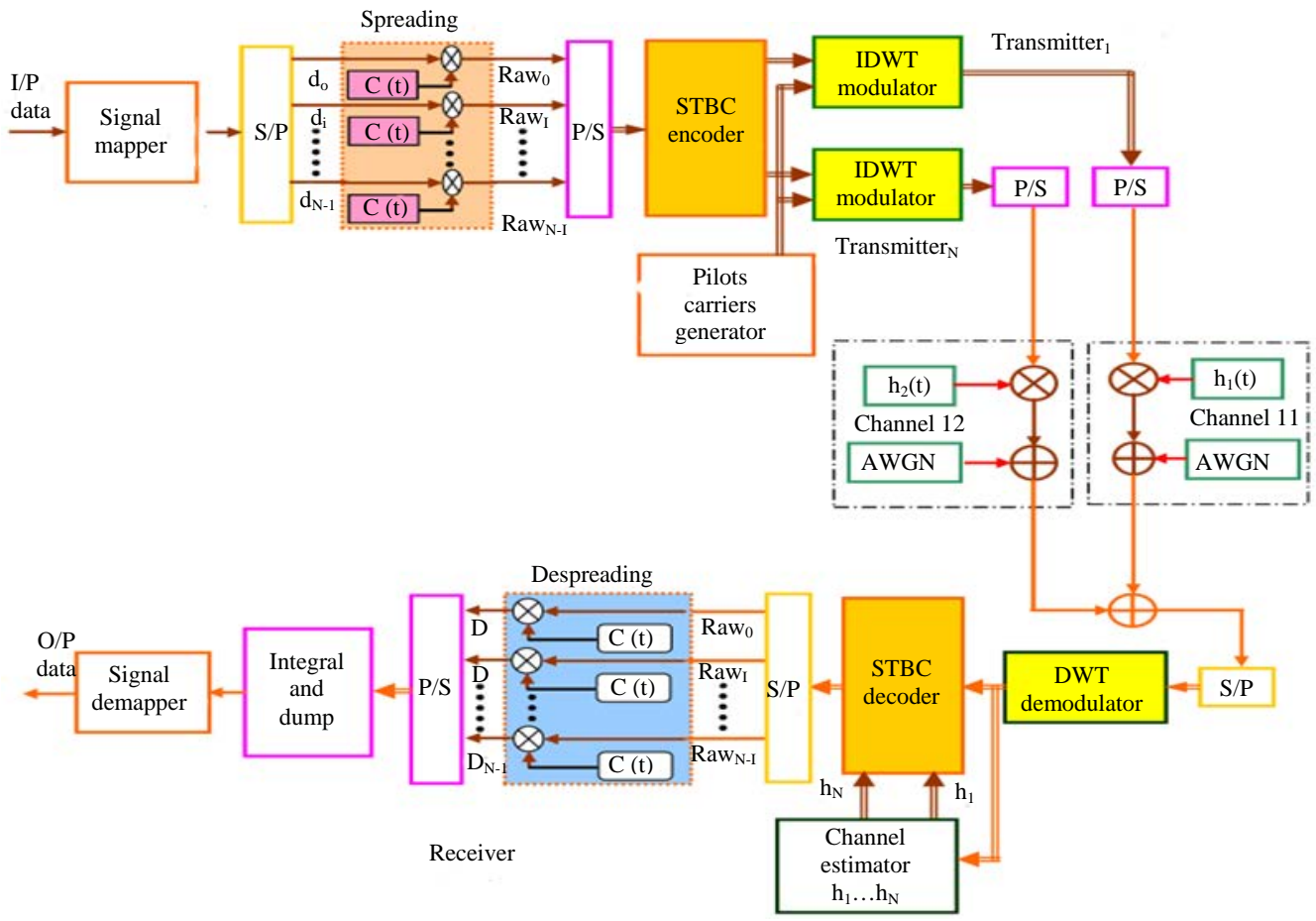

Fig. 1: Proposed STBC-MC-DS-CDMA system block

Table 1: STBC mapping for four transmit antennas using complex signals

Four transmit antennas (Three transmit antennas)

\begin{tabular}{lllll} 
Time slot & $\mathrm{T}_{\mathrm{x} 1}$ & $\mathrm{~T}_{\mathrm{x} 2}$ & $\mathrm{~T}_{\mathrm{x} 3}$ & $\mathrm{~T}_{\mathrm{x} 4}$ \\
\hline Slot T0 & $\mathrm{S}_{1}$ & $\mathrm{~S}_{2}$ & $\mathrm{~S}_{3}$ & $\mathrm{~S}_{4}$ \\
Slot T1 & $-\mathrm{S}_{2}$ & $\mathrm{~S}_{1}$ & $-\mathrm{S}_{4}$ & $\mathrm{~S}_{3}$ \\
Slot T2 & $-\mathrm{S}_{3}$ & $\mathrm{~S}_{4}$ & $\mathrm{~S}_{1}$ & $-\mathrm{S}_{2}$ \\
Slot T3 & $-\mathrm{S}_{4}$ & $-\mathrm{S}_{3}$ & $\mathrm{~S}_{2}$ & $\mathrm{~S}_{1}$ \\
Slot T4 & $\mathrm{S}^{*}{ }_{1}$ & $\mathrm{~S}^{*}{ }_{2}$ & $\mathrm{~S}^{*}$ & $\mathrm{~S}^{*}{ }^{*}$ \\
Slot T5 & $-\mathrm{S}^{*}{ }_{2}$ & $\mathrm{~S}^{*}{ }_{1}$ & $\mathrm{~S}^{*}$ & $\mathrm{~S}^{*}$ \\
Slot T6 & $-\mathrm{S}^{*}{ }_{3}$ & $\mathrm{~S}^{*}{ }_{4}$ & $\mathrm{~S}^{*}$ & $-\mathrm{S}^{*}$ \\
Slot T7 & $-\mathrm{S}^{*}{ }_{4}$ & $-\mathrm{S}^{*}{ }_{3}$ & $\mathrm{~S}^{*}$ & $\mathrm{~S}^{*}$ \\
\hline
\end{tabular}

used each one to estimate the corresponding channel transfer function $\mathrm{h} 1(\mathrm{t}), \mathrm{h} 2(\mathrm{t}), \mathrm{h} 3(\mathrm{t})$ and $\mathrm{h} 4(\mathrm{t})$ using the following equation:

$$
\mathrm{H}_{\text {1or } 2,3,4}(\mathrm{k})=\frac{\text { Received training sample }_{\text {or } 2,3,4}(\mathrm{k})}{\text { Transmitted training sample }(\mathrm{k})_{1}}, \ldots, \mathrm{k}=0,1, \ldots, \mathrm{N} / 2-1
$$

After this process $\mathrm{h} 1(\mathrm{t}), \mathrm{h} 2(\mathrm{t}), \mathrm{h} 3(\mathrm{t})$ and $\mathrm{h} 4(\mathrm{t})$ are estimated.

Spreading codes (gold codes): The gold sequence set is best known binary sequences which have good correlation values. The gold sequences is generated from a distinguish pair of m-sequences, $\mathrm{x}$ and $\mathrm{y}$ having same length Q. Each Gold sequence set is generated by a modulo-2 sum (XOR) of $\mathrm{x}$ and cyclic shifts of $\mathrm{y}$. The entire set of gold sequences having a period of $\mathrm{Q}$ is given by:

$$
\mathrm{Sg}=\left\{\mathrm{x}, \mathrm{y}, \mathrm{x} \oplus \mathrm{y}, \mathrm{x} \oplus \mathrm{T}^{-1} \mathrm{y}, \mathrm{x} \oplus \mathrm{T}^{-2} \mathrm{y}, . ., \mathrm{x} \oplus \mathrm{T}^{-(Q-1)} \mathrm{y}\right\}
$$

where, $\mathrm{T}^{-\mathrm{q}} \mathrm{y}$ for $\mathrm{q}=1,2, \ldots, \mathrm{Q}-1$, represents a cyclic shift of y by q chip intervals and the symbol $\oplus$ represents modulo-2 addition (Ahmadinejad and Talebi, 2016). The system employs a set of spreading sequences for each user. Each user in turn, applies a different sequence from that set to each of his sub-carriers. These sequences are Pseudo Noise (PN) sequences. A binary sequence (with elements $\{0,1\}$ ) is mapped into a corresponding sequence of positive and negative pulses according to the relation:

$$
P_{i}(t)=\left(2 b_{i}-1\right) p(t-i t)
$$

where, $p_{i}(t)$ is the pulse corresponding to the element $b_{i}$ in the sequence with elements $\{0,1\}$. Spreading sequences can be bipolar or poly-phase. The different sequences which can be used as spreading sequences are gold sequences, Kasami sequences, poly phase sequences, four phase sequences and Mutually Orthogonal Complementary sets of Sequences (MOCS).

Signal model: Multiple accesses can be generated by using any spreading code to each user in the CDMA system. The used of DWT in multicarrier direct sequence CDMA is like using DWT in OFDM system (Bagadi et al., 2016). The DWT symbols duration consider of T, bandwidth $20 \mathrm{MHz}$, spanning Ns $=256$ 
samples (equivalent to 256 sub-carriers of OFDM) to be transmitted in different sub-bands (Zakaria and Ruyet, 2014). The output of this operation can express in this Eq. 4:

$$
y(k, n)=\sum_{M=1}^{4} H_{M}(k) S_{M}(k, n)+N(k, n)
$$

where, $\mathrm{N}(\mathrm{k}, \mathrm{n})(2 \times 1)$ is a complex-valued additive white Gaussian noise vector with entities of zero mean and variance $\sigma_{\mathrm{z}}^{2} \mathrm{H}(\mathrm{k})(4 \times 1)$ denotes the channel frequency response according to the ITU channel models (Zakaria and Ruyet, 2014). The channel assumed that certain frequency band keeps constant within the time interval of N OFDM symbols. The (i, j)th element of H(k) is given by:

$$
[\mathrm{H}(\mathrm{k})]_{\mathrm{i}, \mathrm{j}}=\mathrm{X} \sum_{\mathrm{l} \geq 0} \sum_{\mathrm{m} \geq 0} \alpha_{\mathrm{m}, \mathrm{l}^{\mathrm{e}}}^{\mathrm{i}, \mathrm{j} 2 \pi k \Delta f\left(\mathrm{~T}_{1}^{\mathrm{i}, j}+\tau_{\mathrm{m}, \mathrm{i}, \mathrm{l}}^{\mathrm{i}, \mathrm{l}}\right)}
$$

where, $\Delta \mathrm{f}$ is the frequency difference between two adjacent subcarriers $\alpha_{m, 1}^{i, j}$ is the gain of multipath to cluster $l$ and path $m$ between the jth transmit antenna and the ith receive antenna; the lth cluster arrives at $\mathrm{T}_{1}^{\mathrm{i}, \mathrm{j}}$ and its kth path arrives at $\tau_{\mathrm{m}, \mathrm{i}}^{\mathrm{i}, \mathrm{j}} \mathrm{X}$ represents the shadowing of Rayleigh's distributed random variable, i.e., $20 \log _{10} \mathrm{X} \propto$ $\mathrm{N}\left(0, \sigma_{\mathrm{x}}^{2}\right)$ while the total energy contained in the terms $\alpha_{\mathrm{m}, \mathrm{l}}^{\mathrm{i}, \mathrm{j}} \forall \mathrm{m}$, l for each couple (i,j) is normalized to unity for each channel realization. For simplicity of notation, the indices of $\mathrm{k}$ and $\mathrm{n}$ and denote hi, $\mathrm{j}=[\mathrm{H}(\mathrm{k})]_{\mathrm{i}, \mathrm{j}}$ and $\mathrm{ci}, \mathrm{j}=$ $\sum_{1 \geq 0} \sum_{m \geq 0} \alpha_{m, l^{\mathrm{e}}}^{\mathrm{i}, \mathrm{j} 2 \pi k \Delta f}\left(\mathrm{~T}_{\mathrm{i}}^{\mathrm{i}, \mathrm{j}}+\tau_{\mathrm{m} . \mathrm{l}}^{\mathrm{i}, \mathrm{j}}\right)$, respectively. Thus, it shows that:

$$
h_{i, j}=X_{i, j}
$$

When the Alamouti coding is applied, the system is equivalent to independent single-input single-output systems defined as (Simsir and Taspinar, 2017; Nagaradjane et al., 2013):

$$
u_{j}=\phi d_{j}+\varsigma_{j}
$$

Where, $\phi=\sum_{\mathrm{i}=1}^{2} \sum_{\mathrm{j}=1}^{2}\left|\mathrm{~h}_{\mathrm{i}, \mathrm{j}}\right|^{2}$, dj denote original symbols before dispreading and $\mathrm{j}_{\mathrm{j}}$ is an equivalent complex Gaussian random variable with zero mean and variance $\phi \sigma_{z}^{2}$ from (5), it can rewrite $\phi=\sum_{i=1}^{2} \sum_{i=1}^{2}\left|c_{i, j}\right|^{2}$. Therefore, the output Signal to Noise Ratio (SNR) by Shi et al. (2009) can be expressed as:

$$
\gamma=\frac{\rho_{\mathrm{s}}}{\sigma_{\mathrm{z}}^{2}} X^{2} \chi
$$

Where $\mathrm{x}=\sum_{\mathrm{i}=1}^{2} \sum_{\mathrm{j}=1}^{2}\left|\mathrm{c}_{\mathrm{i}, \mathrm{j}}\right|^{2}$ and $\rho_{\mathrm{s}}$ denotes the averaged power of transmitted symbols. Therefore, the SNR (S/N), at the output of receiver can be written as:

$$
\left(\operatorname{SNR} \frac{\mathrm{E}\left\{\mathrm{X}_{\mathrm{I}} \mid \alpha_{\mathrm{I}}^{(1)}\right\}^{2}}{\operatorname{VAR}\left\{\mathrm{X}_{\mathrm{I}} \mid \alpha_{\mathrm{I}}^{(1)}\right\}}\right)=\mathrm{N}^{2} \mathrm{Ec} \gamma
$$

For BPSK signaling, the evaluation of average BER for different scenarios can be approximately achieved by:

$$
\begin{aligned}
& \mathrm{P}_{\mathrm{BER}}^{\mathrm{su}}=\int_{0}^{\infty} \mathrm{Q}\left\{\frac{\mathrm{E}\left\{\xi_{\mathrm{u}}\right\}}{\sqrt{\operatorname{VAR}\left(\xi_{\mathrm{u}}\right)}}\right\} \mathrm{f}\left(\alpha^{(1)}\right) \frac{\text { Mfolds }}{\mathrm{d \alpha _{1 } ^ { ( 1 ) } , \ldots , \mathrm { d } \alpha _ { \mathrm { M } } ^ { ( 1 ) }}=} \\
& \int_{0}^{\infty} \mathrm{Q}\left(\sqrt{\mathrm{N}^{2} \mathrm{E}_{\mathrm{C \gamma su}}}\right) \mathrm{f}_{\gamma}\left(\gamma_{\mathrm{su}}\right) \mathrm{d}\left(\delta_{\mathrm{su}}\right)
\end{aligned}
$$

The $\mathrm{Q}(\mathrm{x})$ in (10) is the Gaussian Q-function. Hereafter, the average BER of an STBC BASED MC-DSCDMA system over fading channel can be calculated from (Abdul-Rahaim, 2015a, b).

Propagation loss models in STBC based MC-DSCDMA system: Choosing the right propagation models of wireless channel is critical in the design of any wireless networks. These models are used to predict the path loss between the transmitter and the receiver which represents the combined effects on signal level attenuation due to the free space propagation, reflection, diffraction and scattering, etc. There are a number of empirical and statistical propagation models for outdoor and indoor environments, these models include the "Okumura-Hata Model, COST 231-Hata Model, COST 231 WalfishIkegami Model, Erceg Model, Stanford University Interim (SUI) Channel Models and ITU Path Loss Models” and the define of each one of them shown by Bagadi et al. (2016). Each propagation model is defined for a specific environment. In this study, ITU path loss models will be use.

ITU path loss models: A set of commonly used experimental channel models and identified in the ITU-R Recommendation M.1225. Recommendation defines three different environments to test: "Indoor office, outdoor-to indoor pedestrian and vehicular". Since, the spread of the delay can vary significantly, identifies the recommendation of the spread of two different delays for each test environment: low delay spread and medium delay spread in all there are 6 cases. Each case of these have a specified multipath tap delay profile. The multipath have different number of components in each model. Table 2 show the list the specified parameters of [M.1225] in low delay spread like "ITU Channel Model for Indoor Office test, ITU Channel Model for Outdoor to Indoor and Pedestrian Test and ITU Channel Model for Vehicular Environment Test” (Anonymous, 2011).

Channel estimation of STBC based MC-DS-CDMA system: Since, the radio channel is frequency selective 
J. Eng. Applied Sci., 14 (Special Issue 9): 10740-10757, 2019

Table 2: ITU channel models for indoor office and outdoor to indoor and pedestrian and vehicular environment

ITU Channel Model for indoor ITU Channel Model for outdoor to indoor ITU Channel Model for vehicular office test and pedestrian test environment test

\begin{tabular}{|c|c|c|c|c|c|c|}
\hline \multirow[b]{2}{*}{ Path \# } & \multicolumn{2}{|c|}{ 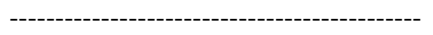 } & & \\
\hline & Delay (nsec) & Power (dB) & Delay in nsec & Power (dB) & Delay (nsec) & Power (dB) \\
\hline 1 & 0 & 0 & 0 & 0.0 & 0 & 0.0 \\
\hline 2 & 50 & -3.00 & 110 & -9.7 & 310 & -1.0 \\
\hline 3 & 110 & -10.0 & 190 & -19.2 & 710 & -9.0 \\
\hline 4 & 170 & -18.0 & 410 & -22.8 & 1090 & -10.0 \\
\hline 5 & 290 & -26.0 & - & - & 1730 & -15.0 \\
\hline$\underline{6}$ & 310 & -32.0 & - & - & 2510 & -20.0 \\
\hline
\end{tabular}

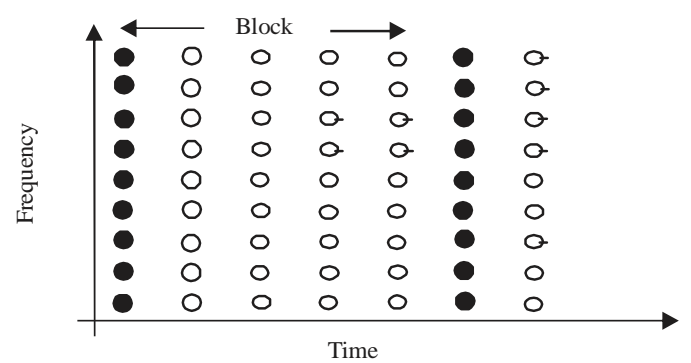

Fig. 2: Block-type pilot channel estimation

and time varying for mobile communications system and dynamic estimation of channel is necessary to retrieve STBC-based MC-DS-CDMA signal in the receiver side (Dawood et al., 2015a, b). In block-type pilot-based channel estimation as shown in Fig. 2, STBC based MC-DS-CDMA channel estimation symbols are transmitted periodically and all subcarriers are used as pilots. The task here is to estimate the channel conditions given the pilot signals and received signals with or without using certain knowledge of the channel statistics. The receiver uses the estimated channel conditions to decode the received data inside the block until the next pilot symbol arrives. If the channel is constant during the block, there will be no channel estimation error, since, the pilots are sent at all carriers. For Least Square (LS), the estimate channel frequency response is given by:

$$
\mathrm{H}_{\mathrm{e}}=\mathrm{X}^{-1} \mathrm{Y}
$$

And this estimated channel $\mathrm{H}_{\mathrm{e}}$ is used to $\mathrm{f}$ estimated the approximate transmitted signal $\mathrm{Xe}(\mathrm{k})$ :

$$
\mathrm{X}_{\mathrm{e}}(\mathrm{k})=\frac{\mathrm{Y}(\mathrm{K})}{\mathrm{H}_{\mathrm{e}}(\mathrm{K})}, \mathrm{K}=0,1, \ldots, \mathrm{N}-1
$$

When the channel is slowly fading, channel estimation can be updated within a block using equalizer decision feedback each sub-carrier (Dawood et al., 2015a, b). The comb-type pilot channel estimation has been introduced to satisfy the need for equalization when the channel changes fast, even in one STBC based MC-DS-CDMA block. In comb-type pilot based channel estimation as shown in Fig. 2 for each transmitted symbol, $\mathrm{Np}$ pilot signals are uniformly inserted into $\mathrm{X}(\mathrm{k})$ according to the following Eq. 13:

$$
X(k)=X(m L+l)=\left\{\begin{array}{c}
X_{p}(m), l=0 \\
\text { inf. data, l=1,.., L- } 1
\end{array}\right.
$$

Where:

$\mathrm{L} \quad=$ Number of carriers

$\mathrm{N}_{\mathrm{p}}$ and $\mathrm{X}_{\mathrm{p}}(\mathrm{m})=$ The $\mathrm{mth}$ pilot carrier

$\mathrm{H}_{\mathrm{p}}(\mathrm{k}) \quad=$ The channel frequency response at the pilot sub-carriers

The estimation of the channel at pilot sub-carriers based on LS estimation is given by:

$$
H_{e}(k)=\frac{Y_{p}(k)}{X_{p}(k)}, k=0,1, \ldots, N_{p}-1
$$

where, $Y_{p}(k)$ and $X_{p}(k)$ are the received and transmitted signals at kth pilot subcarrier, respectively. In comb-type pilot based channel estimation an efficient interpolation technique is necessary in order to estimate channel at data sub-carriers by using the channel information at pilot sub-carriers.

\section{RESULTS AND DISCUSSION}

Proposed systems simulation results in ITU channel models: The simulation of the proposed STBC DWT-MC-DS-CDMA systems was done by MATLAB R2015a. The BER performance of the MC-DS-CDMA systems had been calculated and computed in AWGN and flat fading channel. The carrier frequency used is $5.8 \mathrm{GHz}$ for fixed and mobile STBC-MC-DS-CDMA system with three values of fd " $10.7 \mathrm{~Hz}$ with speed $2 \mathrm{kmh}^{-1}, 241.7 \mathrm{~Hz}$ with speed $45 \mathrm{kmh}^{-1}$ and $537 \mathrm{~Hz}$ with speed $100 \mathrm{kmh}^{-1}$ ”. Table 3 shows the parameters of the system used in the simulation.

The proposed STBC-MC-DS-CDMA DWT and FFT systems had been simulated using MATLAB R2015a in three different test environments: "indoor office, outdoor-to indoor pedestrian and vehicular according to ITU-R Recommendation” as shown in Table 2. The 
J. Eng. Applied Sci., 14 (Special Issue 9): 10740-10757, 2019

Table 3: Simulation parameter

\begin{tabular}{|c|c|c|c|c|c|}
\hline Parameters & Fixed MC-DS-CDMA & \multicolumn{4}{|c|}{ Mobile MC-DS-CDMA scalable } \\
\hline$\overline{\text { FFT or DWT size }}$ & 256 & 128 & 512 & 1024 & 2048 \\
\hline Number of used data subcarriers & 96 & 64 & 180 & 360 & 720 \\
\hline Modulation types & BPSK & & & & \\
\hline Cyclic prefix or guard time (Tg/Tb) & $1 / 16$ & & & & \\
\hline Channel bandwidth (MHz) & 20 & 20 & 20 & 20 & 20 \\
\hline Channel types & ITU channel models & & & & \\
\hline Carrier frequency fc & $5.8 \mathrm{GHz}$ & & & & \\
\hline
\end{tabular}

Carrier frequency fc

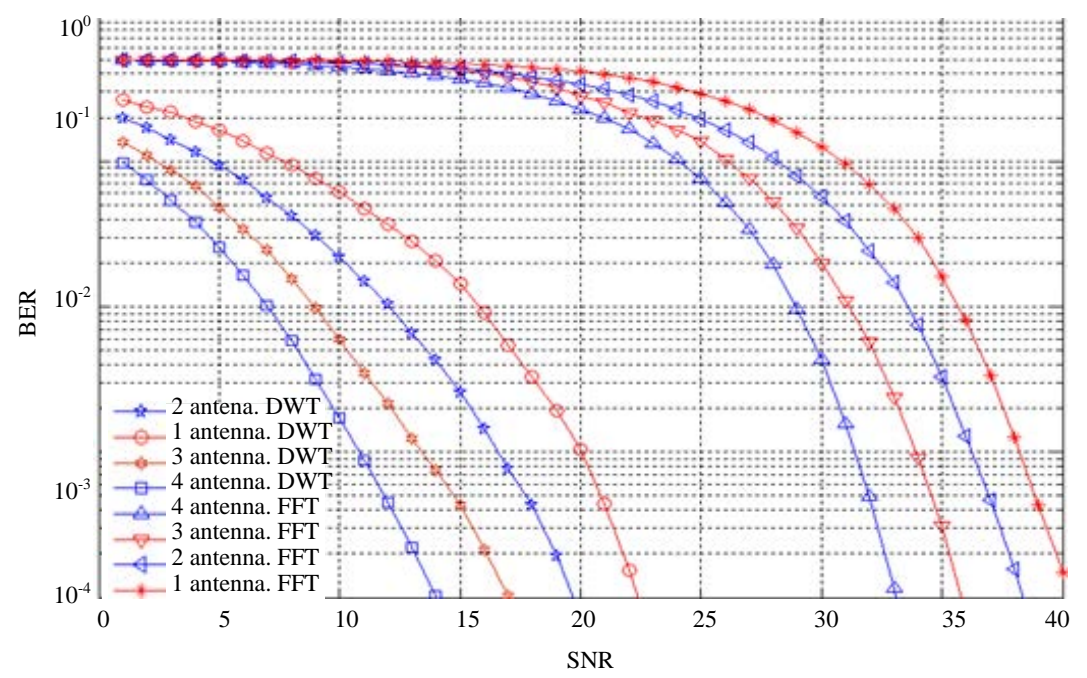

Fig. 3: SNR versus BER to fixed STBC-MC-DS-CDMA-256 subcarriers in ITU indoor office channel model

BER performance of the proposed STBC based MC-DS-CDMA system considered in different type of channel models mentioned above, "the AWGN channel, the flat fading channel and multipath fading channel”. The other parameters the same in Table 3 above to simulate the proposed STBC based MC-DS-CDMA systems.

Four antennas at the transmitter and one antenna at the receiver are sufficiently spaced such that the channels between different transmit-receive antenna pairs are independent. Each channel is frequency-selective with respect to the overall system bandwidth but each subband is assumed to be frequency non-selective with Rayleigh-distributed fade amplitudes. In selective multipath fading channel, many parameters like the influence of the attenuation, delay and maximum Doppler shift of the echo considered to compare the BER performance of the proposed systems. In these cases of channels, the frequency components of the transmitted signal are affected by uncorrelated changes where the parameters of the channel in this case corresponding to multipath, the six paths are chosen, the Line of Sight (LOS) is one of them and the five others paths are the reflected paths.

ITU indoor office channel model: The model of this channel was shown in Table 2 first column. The simulation had been done to all scenario of STBC-MC-
DS-CDMA with DWT and FFT and the simulation result shown in Fig. 3-6. In fixed STBC-MC-DS-CDMA system, it is noted that the proposed STBC-MC-DSCDMA based on DWT performs better than STBC-MCDS-CDMA based on FFT. The SNR at a BER $10^{-4}$ is about $19 \mathrm{~dB}$ for STBC-MC-DS-CDMA based on DWT with 4 antennas and about $37 \mathrm{~dB}$ for STBC-MC-DSCDMA based on FFT with also using 4 antennas. A gain about $18 \mathrm{~dB}$ has been achieved by using DWT way over using FFT system and this value is much need in the communications systems to save transmitted power and increase data rate.

For mobile STBC-MC-DS-CDMA system, the results of 128 and 1024 subcarriers (the size of DWT or FFT) only have been found in three cases of MDS $(10.7 \mathrm{~Hz}$, $241.7 \mathrm{~Hz}$ and $537 \mathrm{~Hz}$ ). It can be seen from Fig. 4 (for small MDS $=10.7 \mathrm{~Hz}$ ) that the proposed STBC-MC-DSCDMA based on DWT still performs better than STBC-MC-DS-CDMA based on FFT and the system of STBC still gives good results for small and large subcarriers (128 and 1024, respectively). The SNR at a BER $10^{-4}$ is about $12 \mathrm{~dB}$ for 4 antennas in proposed system of 128 and about $17 \mathrm{~dB}$ in 1024 while it's not reach the desired value in STBC-MC-DS-CDMA based on FFT with 128 and 1024 subcarriers. In addition, a wide improvement span is obtained for all values of SNR in these systems. It can be seen from Fig. 5a and 6a for 


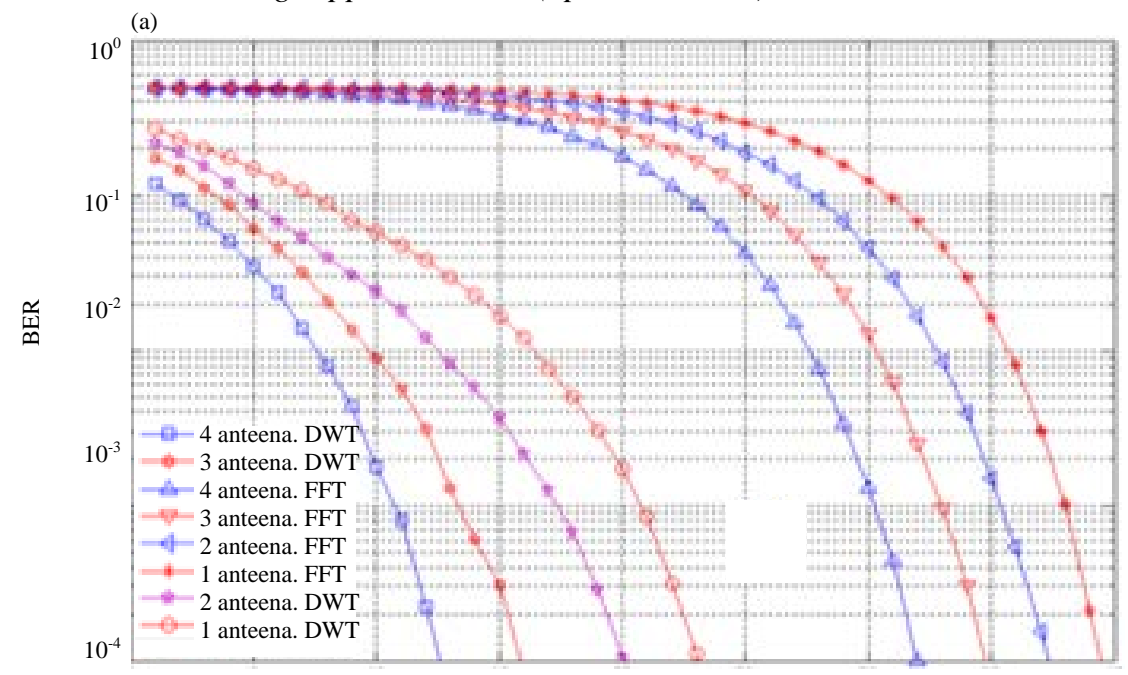

(b)

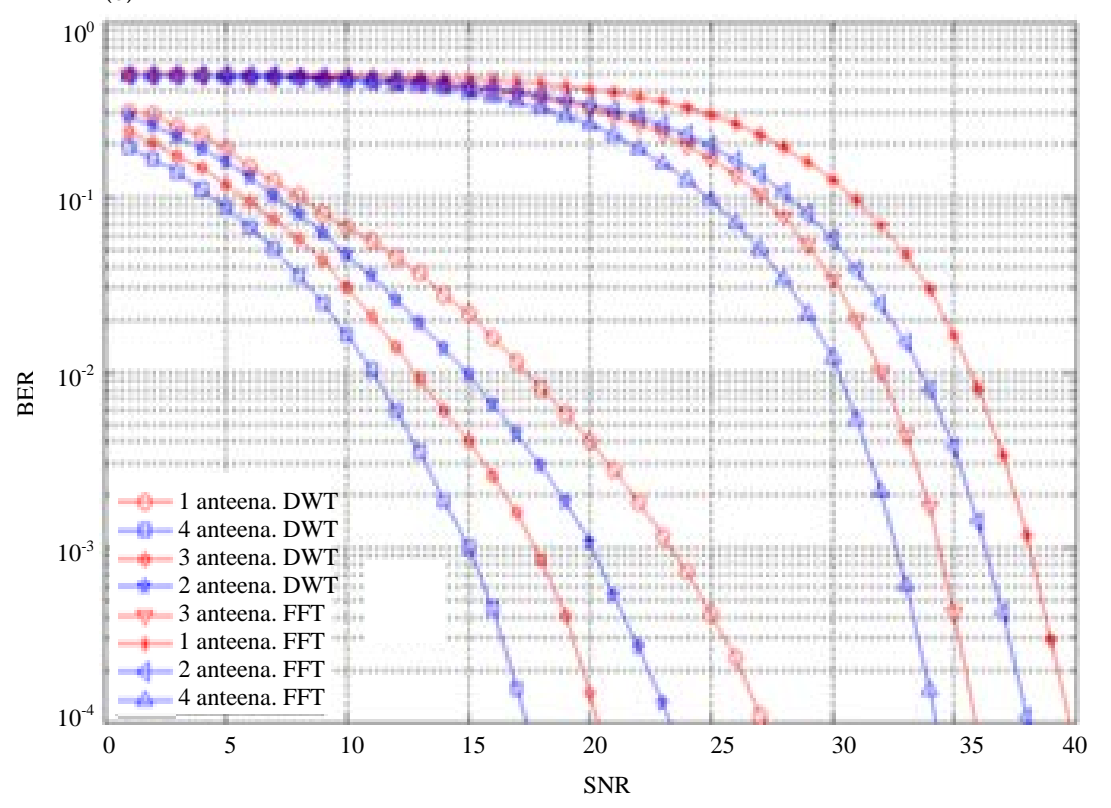

Fig. 4: SNR versus BER to mobile STBC-MC-DS-CDMA ITU indoor office channel model-MDS =10.7Hz (a)-128 subcarriers and (b)-1024 subcarriers

$241.7 \mathrm{~Hz}$ and Fig. (5b) and (6b) for $537 \mathrm{~Hz}$ that the STBC-MC-DS-CDMA based on DWT is performing better than the STBC-MC-DS-CDMA based on FFT but without STBC, MC-DS-CDMA based on DWT is better because of the effect of STBC is eliminated in high Doppler frequency larger than $50 \mathrm{~Hz}$ and the same for other systems.

ITU Channel Model for outdoor to indoor and pedestrian test: In this section, the results of ITU Channel Model for outdoor to indoor and pedestrian test will be simulated according to the Table 2 second column. For fixed proposed STBC-MC-DS-CDMA system, it is clear from Fig. 7 that BER performance of STBC-MC-
DS-CDMA based on DWT is better than STBC-MC-DSCDMA based on FFT system. The SNR at a BER $=10^{-4}$ is about $21 \mathrm{~dB}$ for 4 antennas at proposed system and cannot been reached for STBC-MC-DS-CDMA based on FFT system and this will give losses in the gain about $20 \mathrm{~dB}$ for proposed system against STBC-MC-DSCDMA with FFT system when compared with ITU Channel Model for outdoor to indoor and pedestrian test.

Also, for mobile system, the results have simulated only for 128 and 1024 (the size of DWT or FFT) in three cases of MDS $=(10.7,241.7$ and $537 \mathrm{~Hz})$. From Fig. 8-10, it can be seen that the proposed DWT based STBC-MC-DS-CDMA still performs better than FFT 

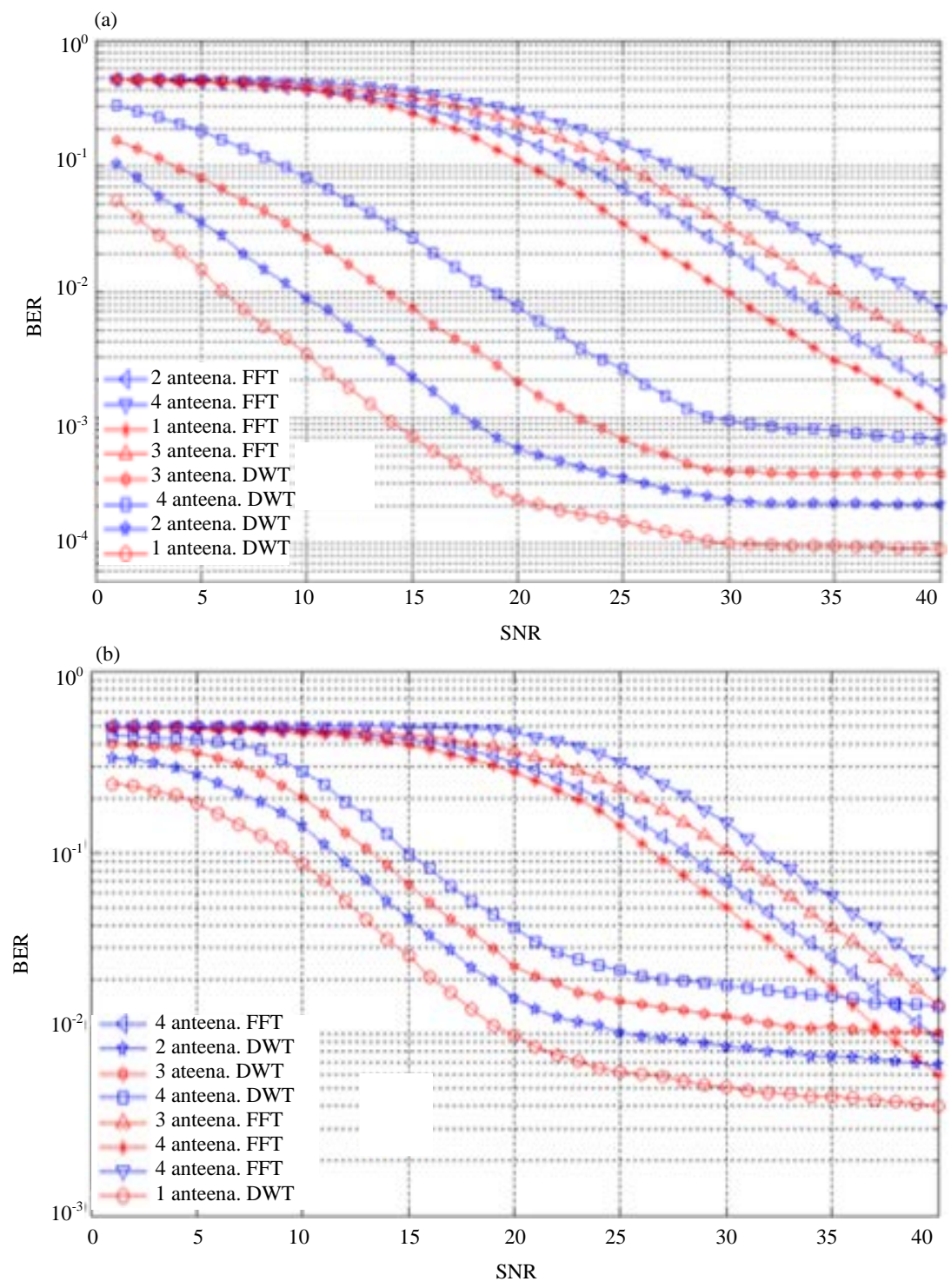

Fig. 5: SNR Versus BER TO Mobile STBC-MC-DS-CDMA-128 subcarriers in ITU indoor office channel model, (a) MDS $=241.7 \mathrm{~Hz}$ and (b) MDS $=537 \mathrm{~Hz}$

based STBC-MC-DS-CDMA and the system of STBC still gives good results. The SNR at a BER $10^{-4}$ is about $15 \mathrm{~dB}$ for 4 antennas at proposed system of 128 and about $19 \mathrm{~dB}$ for 1024 while it is cannot been reach in FFT based STBC-MC-DS-CDMA system of 128 and 1024, this means losses in gain is about $20 \mathrm{~dB}$ for the proposed STBC-MC-DS-CDMA system and cannot been reach for the traditional system in comparison with the ITU Channel Model for outdoor to indoor and pedestrian test at MDS of $10.7 \mathrm{~Hz}$. It can be seen from figures above that the losses will be increased for both systems due to Doppler effect.

ITU Channel Model for vehicular environment test: In this section the results of ITU channel model for vehicular environment test for vehicular test environment will be achieved. In this case the results will be worse than two other channel models because there are six cases with higher relative delay. For fixed MC-DS-CDMA, it can see that the proposed DWT based STBC-MC-DS-CDMA still performs better than FFT based STBC-MC-DS-CDMA. The SNR at a BER $10^{-4}$ is about $21 \mathrm{~dB}$ for 4 antennas at the proposed system and non-at FFT based STBC-MCDS-CDMA.

For mobile system, the effect of MDS will appear and will directly effect on the system of STBC in the case of MDS higher than $50 \mathrm{~Hz}$ and the BER will increase as the Doppler frequency increases in both models. This will lead to results worse than the results of ITU channel model for vehicular environment channel model as 

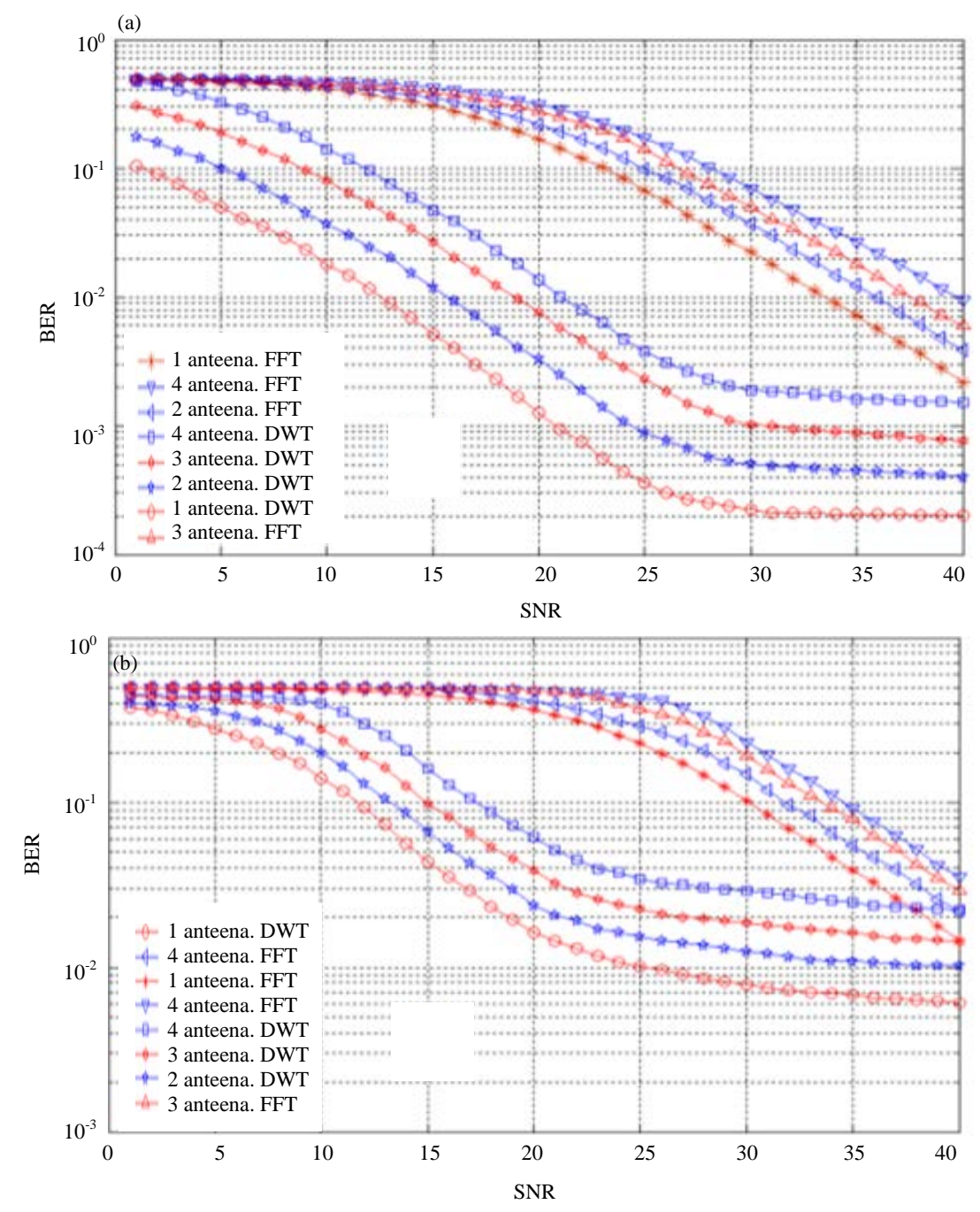

Fig. 6: SNR versus BER to mobile STBC-MC-DS-CDMA-1024 subcarriers ITU indoor office channel model, (a) MDS $=241.7 \mathrm{~Hz}$ and (b) MDS $=537 \mathrm{~Hz}$

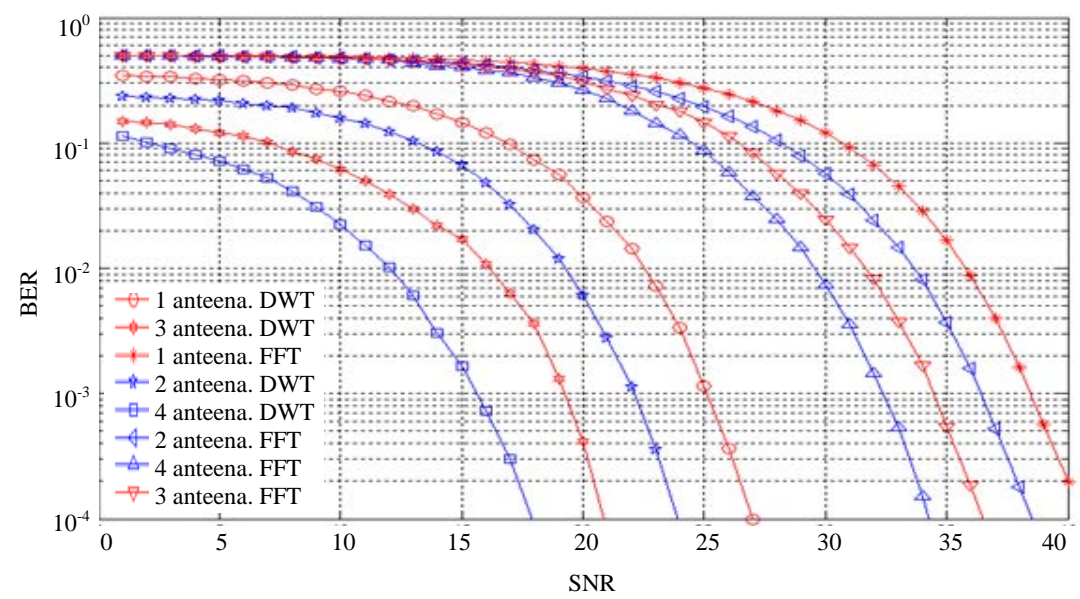

Fig. 7: SNR versus BER to fixed STBC-MC-DS-CDMA-256 subcarriers in ITU Channel Model for outdoor to indoor and pedestrian test 

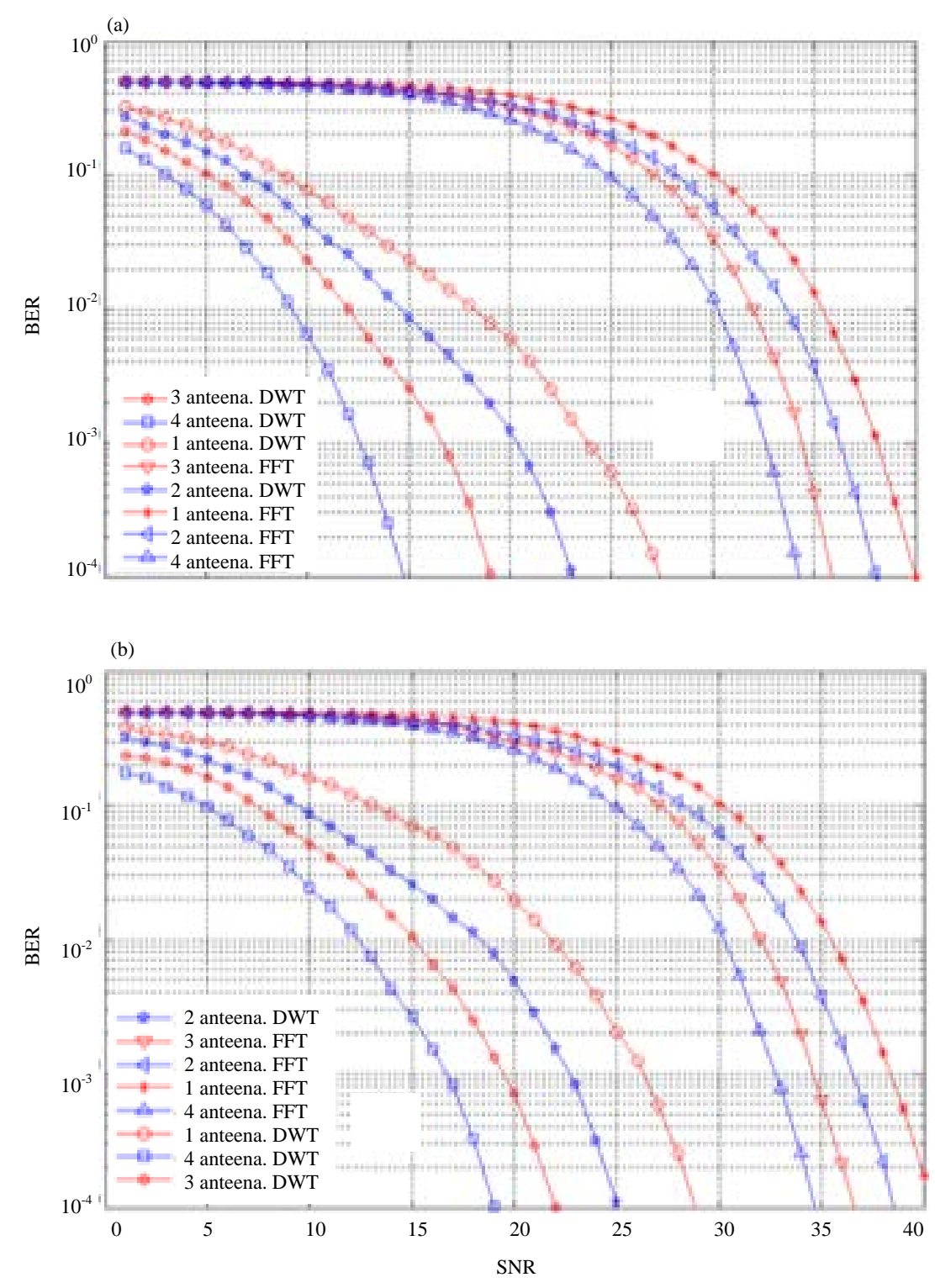

Fig. 8: SNR versus BER to mobile STBC- MC-DS-CDMA- in ITU Channel Model for outdoor to indoor and pedestrian test-MDS $=10.7 \mathrm{~Hz}$, (a) 128 subcarriers and (b) 1024 subcarriers

display in the below figures. All the results present in paper computed after testing the system by transmitted over 10M bits (Fig. 11).

The BER performance of AWGN channel as show from the simulation results is the best of all channels as it has the lowest Bit Error Rate (BER) and power losses with using BPSK modulation schemes. The amount of noise occurs in the BER to this channel type is quite slighter than flat fading channels models (Fig. 12). Simulations proved that the accumulation STBC and DWT improved the bit error rates BER performance than accumulation STBC and FFT in MC-DS-CDMA system. The STBC-MC-DS-CDMA with FFT shows that it requires a longer time adapting to noise and performs poorly to system parameter changes. It can be concluded from the comparison of the performance results of new STBC-MC-DS-CDMA based DWT structure with the STBC-MC-DS-CDMA based FFT that for the same model it gives a robust implementation and still performs better BER performance than FFT in all values of the Doppler frequencies model of flat fading channel. In the case of selective multipath fading channel, the simulation done in three cases of propagation losses model according to MC-DS-CDMA channels models. Therefore, it clear from the simulation results that DWT based STBC-MC-DS-CDMA performs better than FFT based 

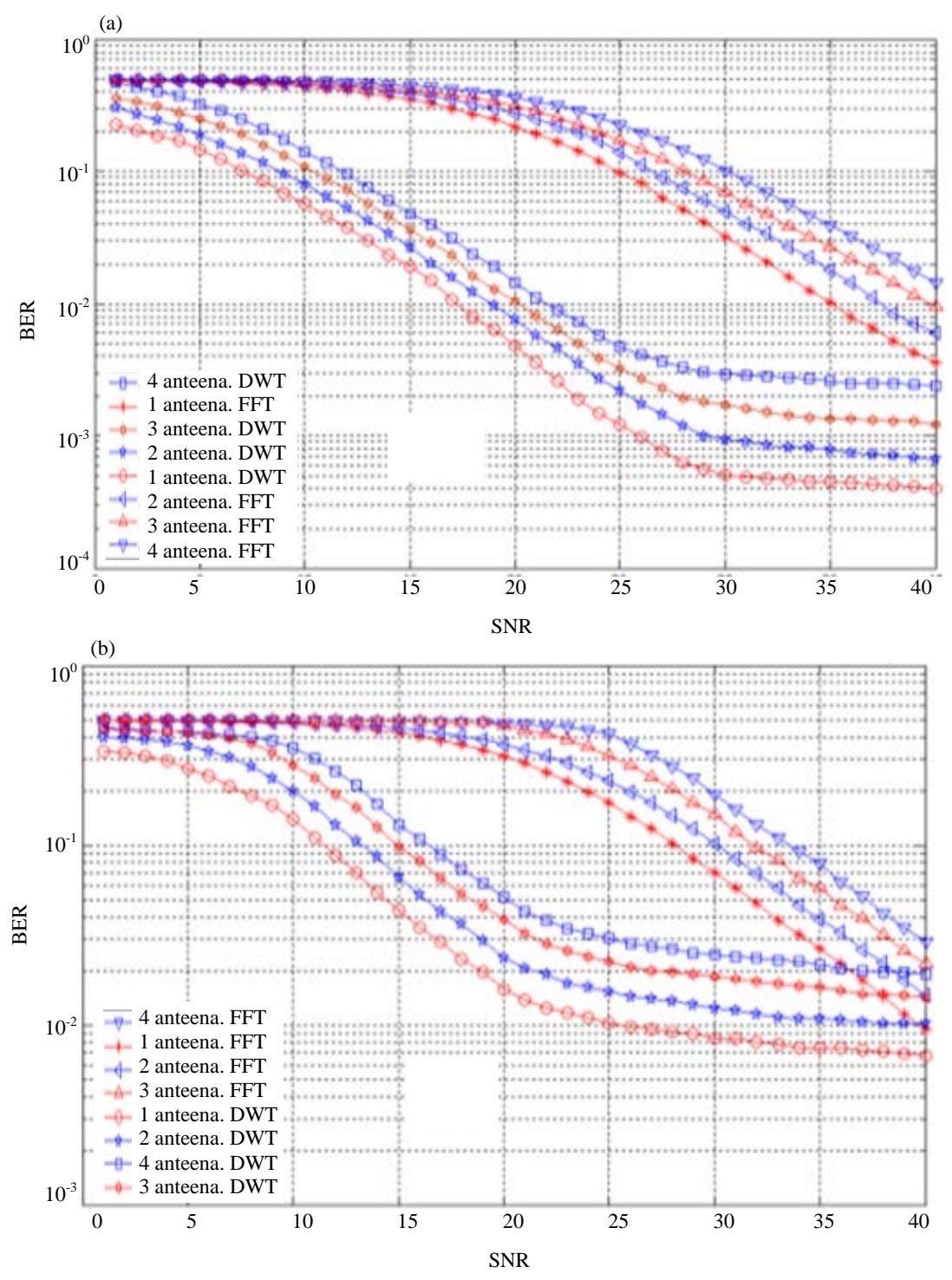

Fig. 9: SNR versus BER to mobile STBC-MC-DS-CDMA in ITU Channel Model for outdoor to indoor and pedestrian test-128 subcarriers, (a) MDS $=241.7 \mathrm{~Hz}$ and (b) MDS $=537 \mathrm{~Hz}$

STBC-MC-DS-CDMA but STBC advantage will be eliminated in high Doppler shift like at 241.7 and $537 \mathrm{~Hz}$ because of each antenna attenuate each other and the losses in ITU Channel Model for vehicular environment test is the worst case among other channel models because of the combined effects of signal level attenuation in vehicular channel model due to the free space propagation, reflection, diffraction and scattering is more than those occurring in the other two channel models (Fig. 13 and 14).

All the results present in this section are summarized in Table 4 for multipath fading channel. These results were computed after testing the system used transfer over $10 \mathrm{M}$ symbols and the tables present only the SNR that get BER of $10^{-4}$.
In the case of multipath fading channel, we can see there are three cases of propagation losses model according to the ITU-R of STBC based MC-DS-CDMA channel. Channel model is helpful in determining the mechanisms that the deployment in an environment that is happening which is useful in the development of the communications system in return. By studying the details of how to deploy a signal from the transmitter to the receiver for a number of pilot sites can be developed in a form highlights the important characteristics due to the indoor environment. In experimental models and all the environmental effects are taken into account implicitly, regardless of whether they can be identified separately. This is the main feature of these models, due to the adoption of deterministic models on the principles of 

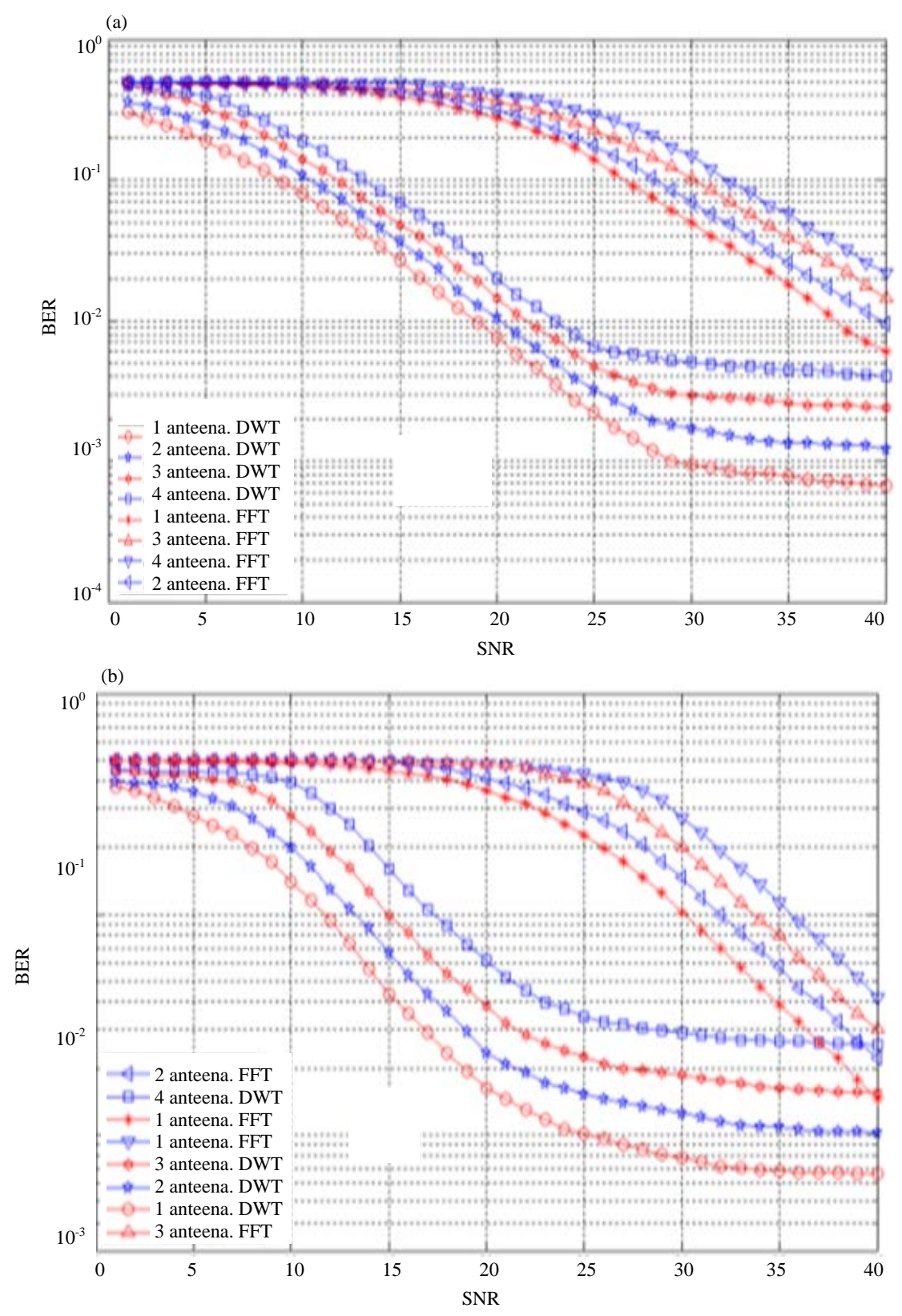

Fig. 10: SNR versus BER to mobile STBC-MC-DS-CDMA-1024 subcarriers in ITU channel model for outdoor to indoor and pedestrian test, (a) MDS $=241.7 \mathrm{~Hz}$ and (b) $\mathrm{MDS}=537 \mathrm{~Hz}$

physics that can be applied to different environments without affecting the accuracy. Propagation within a building (indoor) is yet another problem of interest and has a source within the building. Indoor propagation varies greatly with the type of buildings and the position of access points within the building how far from wall how high compare to obstructions and furniture and is different when signal comes from the outside because the signal will affect by indoor multipaths and outdoor multipaths. Vehicle loss given the mobile nature of wireless communications, penetration loss into vehicle is important as well. Precise characterization of in-vehicle penetration is difficult as well and varies with type of vehicles, frequency, polarization, antenna placement in the vehicle and direction of incidence. Therefore, we can see from Table 4 that DWT performs better than FFT but STBC is eliminated in high Doppler shift like at 241.7 and $537 \mathrm{~Hz}$ because of each antenna attenuate each other and the losses in vehicular channel model is the worst case among other channel models because of the 
J. Eng. Applied Sci., 14 (Special Issue 9): 10740-10757, 2019

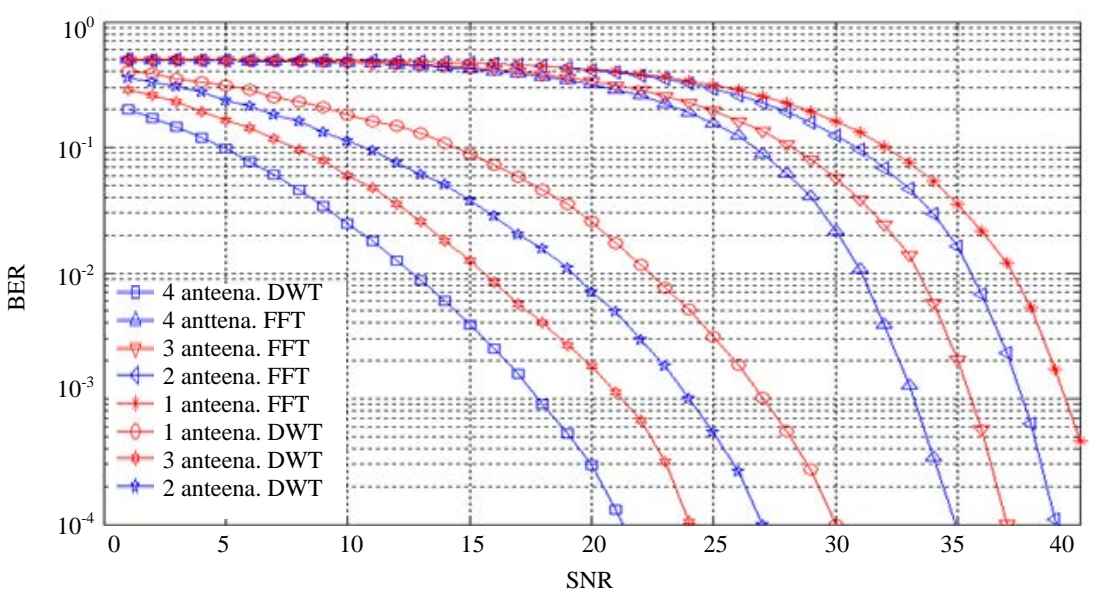

Fig. 11: SNR versus BER to fixed STBC-MC-DS-CDMA-256 subcarriers in ITU CHANNEL MODEL FOR VEHICULAR environment test
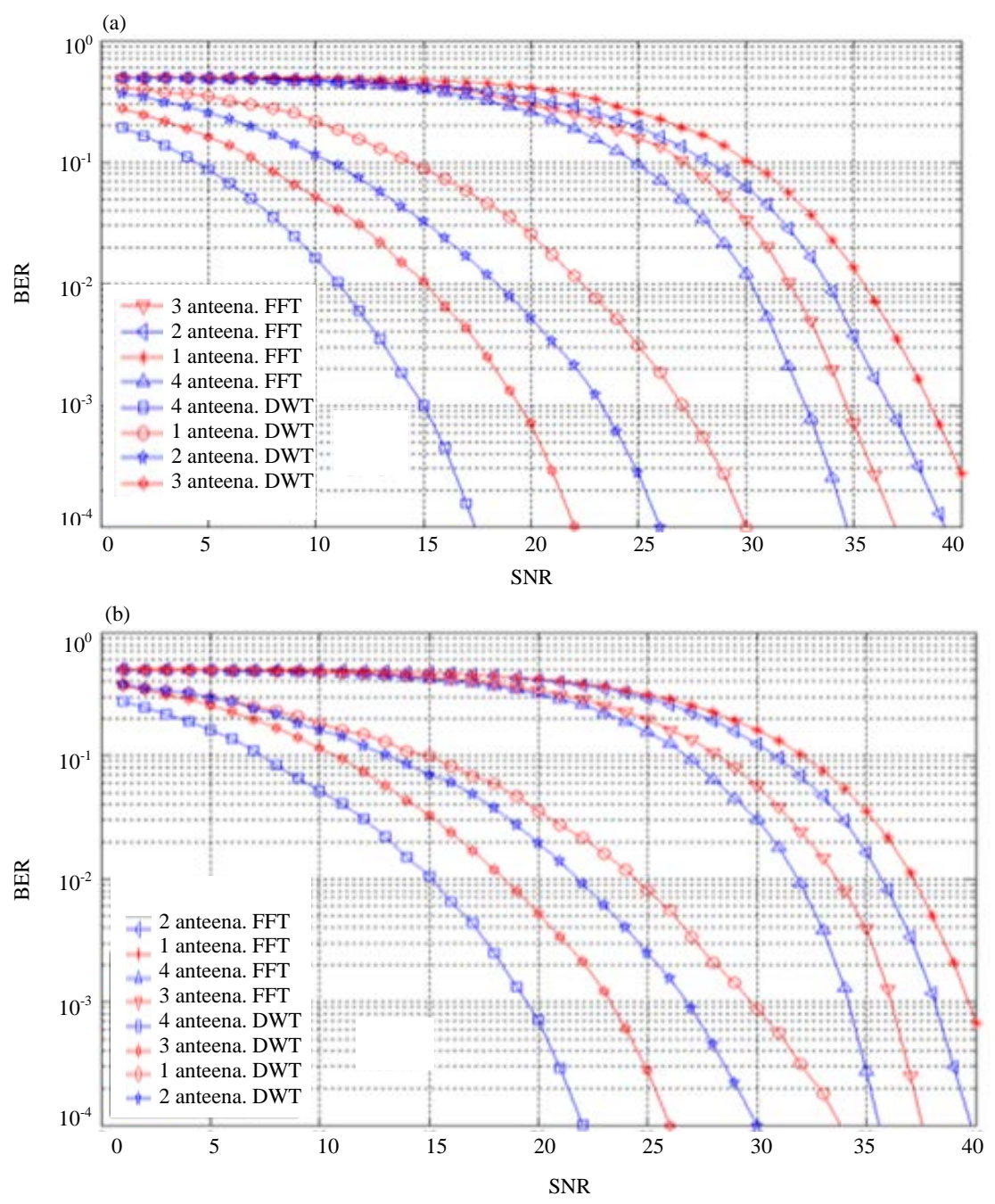

Fig. 12: SNR versus BER to mobile STBC-MC-DS-CDMA-in ITU channel model for vehicular environment test-MDS $=10.7 \mathrm{~Hz}$, (a) 128 subcarriers and (b) 1024 subcarriers 
J. Eng. Applied Sci., 14 (Special Issue 9): 10740-10757, 2019

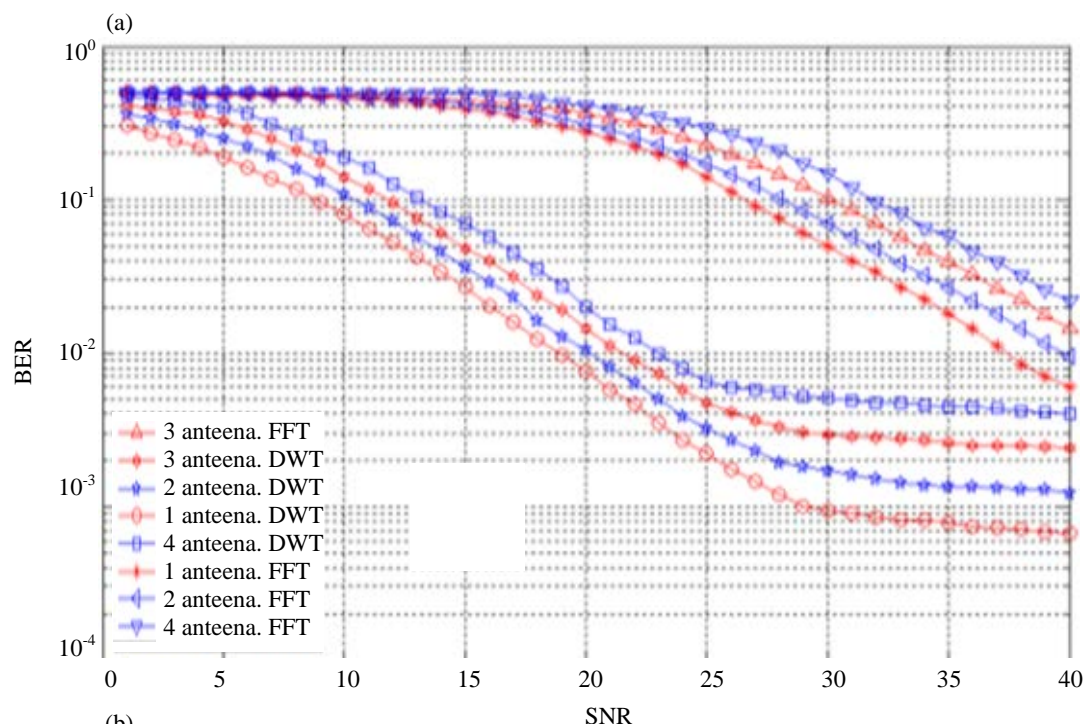

(b)

SNR

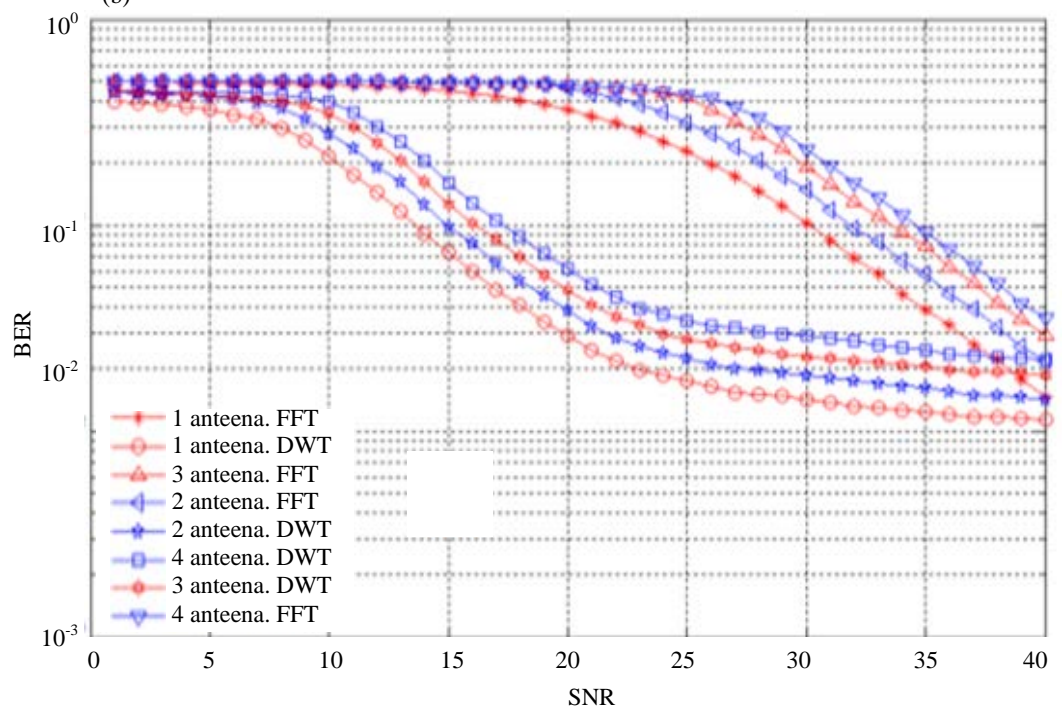

Fig. 13: SNR versus BER to mobile STBC-MC-DS-CDMA-128 subcarriers in ITU channel model for vehicular environment test, (a) MDS $=241.7 \mathrm{~Hz}$ and (b) MDS $=537 \mathrm{~Hz}$

Table 4: SNR of the system that get BER of $10^{-4}$ for selective fading channel

$$
\text { FFT DWT }
$$

\begin{tabular}{|c|c|c|c|c|c|c|c|c|c|c|c|}
\hline & \multirow[b]{2}{*}{ Size } & \multirow[b]{2}{*}{ Channel model } & \multirow[b]{2}{*}{$\operatorname{MDS}(\mathrm{Hz})$} & & \\
\hline System type & & & & 1ant. (dB) & 2ant. (dB) & 3ant. (dB) & 4ant. (dB) & 1ant. (dB) & 2ant. (dB) & 3ant. (dB) & 4ant. (dB) \\
\hline \multirow[t]{3}{*}{ Fixed } & 256 & Ind. & - & Non & Non & Non & 37 & 27 & 25 & 21 & 19 \\
\hline & & Out. & - & Non & Non & Non & Non & 32 & 27 & 25 & 21 \\
\hline & & Veh. & - & Non & Non & Non & Non & 38 & 31 & 25 & 21 \\
\hline \multirow[t]{12}{*}{ Mobile } & 128 & Ind. & 10.7 & Non & Non & Non & Non & 30 & 21 & 15 & 12 \\
\hline & & & 241.7 & Non & Non & Non & Non & Non & Non & Non & Non \\
\hline & & & 537 & Non & Non & Non & Non & Non & Non & Non & Non \\
\hline & & Out. & 10.7 & Non & Non & Non & Non & 35 & 30 & 21 & 14 \\
\hline & & & 241.7 & Non & Non & Non & Non & Non & Non & Non & Non \\
\hline & & & 537 & Non & Non & Non & Non & Non & Non & Non & Non \\
\hline & & Veh. & 10.7 & Non & Non & Non & Non & 35 & 30 & 24 & 19 \\
\hline & & & 241.7 & Non & Non & Non & Non & Non & Non & Non & Non \\
\hline & & & 537 & Non & Non & Non & Non & Non & Non & Non & Non \\
\hline & 1024 & Ind. & 10.7 & Non & Non & Non & Non & 32 & 25 & 21 & 17 \\
\hline & & & 241.7 & Non & Non & Non & Non & Non & Non & Non & Non \\
\hline & & & 537 & Non & Non & Non & Non & Non & Non & Non & Non \\
\hline
\end{tabular}


$\underline{\text { Table 4: Continue }}$

\begin{tabular}{|c|c|c|c|c|c|c|c|c|c|c|}
\hline \multirow[b]{2}{*}{ System type } & \multirow[b]{2}{*}{ Channel model } & \multirow[b]{2}{*}{ MDS (Hz) } & \multicolumn{4}{|l|}{ FFT } & \multicolumn{4}{|l|}{ DWT } \\
\hline & & & 1ant. (dB) & 2ant. (dB) & 3ant. (dB) & 4ant. (dB) & 1ant. (dB) & 2ant. (dB) & 3ant. (dB) & 4ant. (dB) \\
\hline & Out. & 10.7 & Non & Non & Non & Non & 35 & 30 & 24 & 19 \\
\hline & & 241.7 & Non & Non & Non & Non & Non & Non & Non & Non \\
\hline & & 537 & Non & Non & Non & Non & Non & Non & Non & Non \\
\hline & Veh. & 10.7 & Non & Non & Non & Non & 39 & 32 & 26 & 21 \\
\hline & & 241.7 & Non & Non & Non & Non & Non & Non & Non & Non \\
\hline & & 537 & Non & Non & Non & Non & Non & Non & Non & Non \\
\hline
\end{tabular}

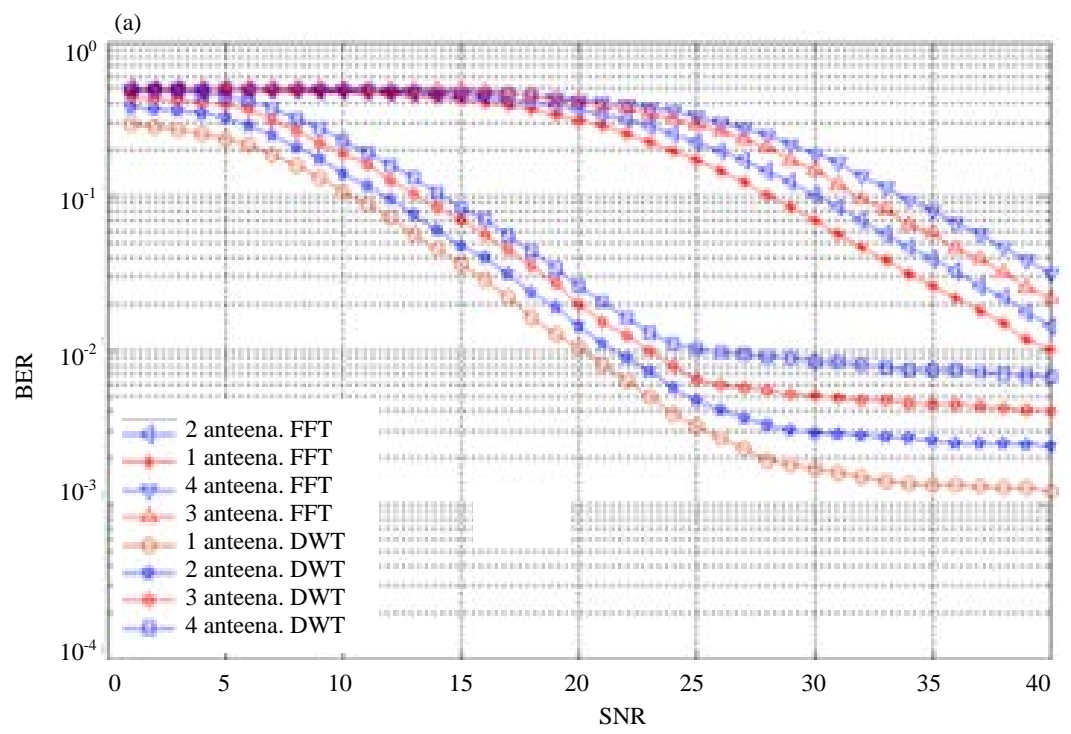

(b)

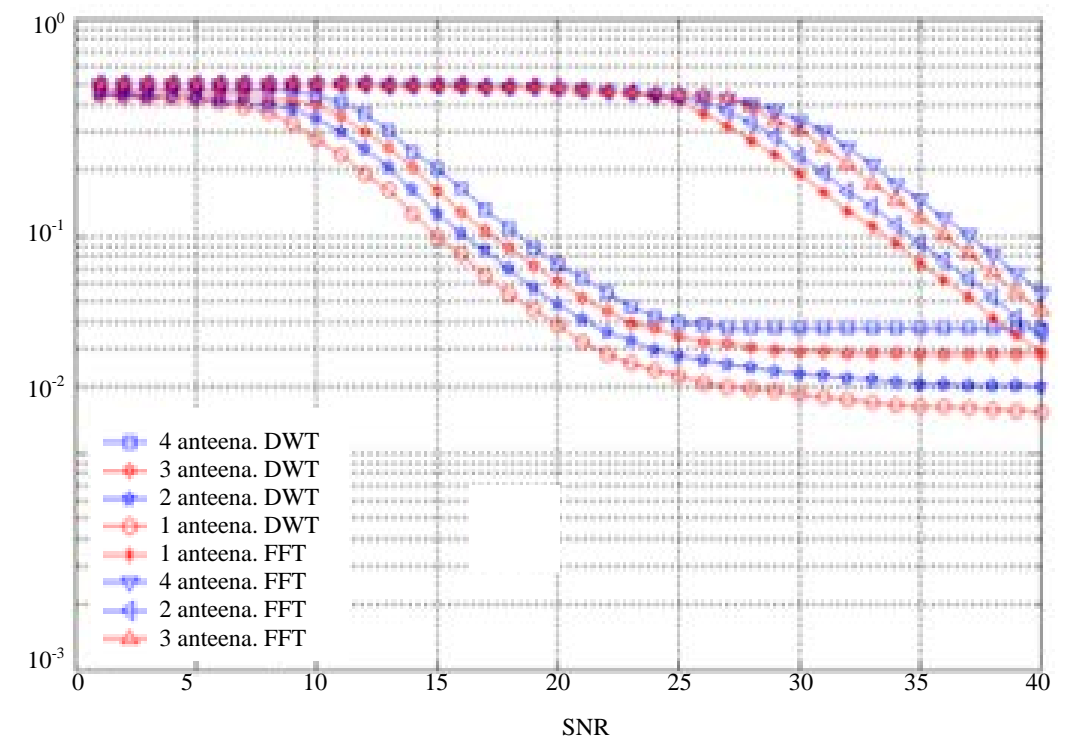

Fig. 14: SNR versus BER to mobile STBC-MC-DS-CDMA-1024 subcarriers in ITU channel model for vehicular environment test, (a) MDS $=241.7 \mathrm{~Hz}$ and (b) MDS $=537 \mathrm{~Hz}$

combined effects of signal level attenuation in vehicular channel model due to the free space propagation, reflection, diffraction and scattering is more than those occurring in the other channel models. It is clear when bit rate increases the bit error rate increase in all systems and DWT system performs better than FFT system because 
DWT helps to reduce the interference and this will help to maintain the orthogonality between adjust subcarriers and provide higher power also STBC provide better performance due to use number of transmitter antennas which resist the effect of fading and reduce the BER when we increase the transmitting of data rate.

\section{CONCLUSION}

This study presents a simulation of the proposed MC-DS-CDMA system base FFT then improved its BER performance and diversity by using space time block coding with two, three and forth antennas. Then DWT have been replacing FFT also to improve BER performance and spectrum efficiency. So, the combination STBC and DWT are given better results, especially with four antennas in transmitter this is a reflection of the fact that the orthogonal base of the wavelets is more significant than the orthogonal bases used in FFT and the orthogonal multiple copies of data due use of STBC. Also, this study focuses on using different multicarrier size (128 and 1024) for mobile wireless communications and 256 for fixed wireless communications. From simulation results when multicarrier size increase BER performance decreased. Also, this study shows the effect of change Doppler frequencies for different value from walking speed $(10.7 \mathrm{~Hz})$ to speed of car in high way $(537 \mathrm{~Hz})$ in mobile wireless communications for the proposed systems and also found that when increase Doppler frequency the BER performance of proposed systems will decreased. So, from all simulation results it's clear that the proposed STBC-MC-DS-CDMA based on DWT was the better system among all system in flat fading channel models at all Doppler frequencies and multicarrier size in fixed and mobile wireless application. In this paper, first the companion of DWT and STBC in MC-DS-CDMA structure has been designed and simulated for the first time. These simulations confirm successful operation and these structures are possibility of implementation. Also, simulation results demonstrate BER performance enhancement that could achieve by combining the DWT and STBC techniques with very little decoding complexity to STBC based MC-DS-CDMA system. As a result, it is clear that proposed STBC-MC-DS-CDMA structure achieves much lower BER in AWGN, flat fading channel and selective multipath fading channel. Therefore, this structure can be considered as an alternative to the conventional STBC based MC-DS-CDMA system. Also, it is well known that the worst scenario to all of the STBC-MC-DS-CDMA systems in terms of performance, occurs when the system parameter is changed. The conventional scheme with FFT shows that it requires a longer time adapting to noise and performs poorly to system parameter changes. It can be concluded from the comparison of the performance of this new structure with the FFT that for the same model it gives a robust implementation. Also, in selective multipath fading channels models, the simulation results are presented by isolating individual propagation effects, to discover which channel parameters have the most significant impact on the performance. In Doppler shift, it is seen that DWT based STBC- MC-DS-CDMA still performs well better than FFT based STBC- MC-DSCDMA but STBC advantage will be eliminated or lost in high Doppler shift above $50 \mathrm{~Hz}$ because of each antenna attenuate each other and the losses in ITU channel model for vehicular environment test is the worst case among other channel models ITU channel model for indoor office test and ITU Channel Model for outdoor to indoor and pedestrian test.

\section{REFERENCES}

Abdul-Rahaim, L.A., 2015a. DWT based WIMAX system with four STBC transmit antennas in multipath fading LTE channels models. Intl. J. Adv. Comput. Technol., 7: 1-12.

Abdul-Rahaim, L.A., 2015b. Enhancement LTE system based on DWT and four STBC transmit antennas in multichannel models. Am. J. Networks Commun., 4: 10-20.

Ahmadinejad, A. and S. Talebi, 2016. Performance evaluation of chaotic spreading sequences in a multi-user MIMO-OFDM system. Phys. Commun., 19: 11-17.

Ali, A.A. and L.A. Abdul-Rahaim, 2008. Discrete wavelets transform based space time block code multicarrier direct sequence CDMA. Proceedings of the IEEE 2008 5th International Multi-Conference on Systems, Signals and Devices, July 20-22, 2008, IEEE, Amman, Jordan, ISBN:978-1-4244-2205-0, pp: 1-4.

Anonymous, 2011. Evolved universal terrestrial radio access (E-UTRA): Physical channels and modulation. ETSI Sophia Antipolis, France. http://www.etsi.org/deliver/etsi_ts/136200_136299/ 136211/10.00.00_60/ts_136211v100000p.pdf

Bagadi, K.P., V. Annepu and S. Das, 2016. Recent trends in multiuser detection techniques for SDMA-OFDM communication system. Phys. Commun., 20: 93-108.

Chen, Y.L. and S.L. Tsao, 2012. A low-latency scanning with association mechanism for real-time communication in mobile WiMAX. IEEE. Trans. Wireless Commun., 11: 3550-3560.

Chisab, R.F. and C.K. Shukla, 2014. The downlink 4G-LTE in fading channel based on the multiwavelet transform. Proceedings of the 2014 International Conference on Computational Intelligence and Communication Networks (CICN), November 14-16, 2014 , I E E E, B hop a l, In d i , ISBN:978-1-4799-6929-6, pp: 233-236. 
Daoud, O., 2012. Performance improvement of wavelet packet transform over fast Fourier transform in multiple-input multiple-output orthogonal frequency division multiplexing systems. IET. Commun., 6: 765-773.

Dawood, S.A., F. Malek, M.S. Anuar and H.A. Rahim, 2015b. Enhancement the performance of OFDM based on multiwavelets using turbo codes. Telkomnika Telecommun. Comput. Electron. Control, 13: 1225-1232.

Dawood, S.A., F. Malek, M.S. Anuar and S.Q. Hadi, 2015a. Discrete multiwavelet critical-sampling transform-based OFDM system over Rayleigh fading channels. Math. Prob. Eng., 2015: 1-10.

Izumi, K., D. Umehara and S. Denno, 2007. Performance evaluation of wavelet OFDM using ASCET. Proceedings of the IEEE International Symposium on Power Line Communications and its Applications ISPLC'07, March 26-28, 2007, IEEE, Pisa, Italy, pp: 246-251.

Kumbasar, V. and O. Kucur, 2012. Performance comparison of wavelet based and conventional OFDM systems in multipath Rayleigh fading channels. Digital Signal Process., 22: 841-846.

Lavanya, R.V. and M. Madheswaran, 2014. Wimax (IEEE $802.16 \mathrm{~m}$ ) system based on space time block code and discrete multiwavelet transform and implementation in FPGA. Telecommun. Syst., 56: 327-334.

Linfoot, S.L., M.K. Ibrahim and M.M. Al-Akaidi, 2007. Orthogonal wavelet division multiplex: An alternative to OFDM. IEEE. Trans. Consum. Electron., 53: 278-284.

Nagaradjane, P., Y.A. Rajan, P. Muralidharan and S.K.V. Sarathy, 2013. Performance of multi-user transmitter preprocessing assisted MIMO system over correlated frequency-selective channels. Phys. Commun., 7: 61-72.
Peng, T., D.R.C. Lamare and A. Schmeink, 2013. Adaptive distributed space-time coding based on adjustable code matrices for cooperative MIMO relaying systems. IEEE. Trans. Commun., 61: 2692-2703.

Shadmand, A., R. Dilmaghani, M. Ghavami and M. Shikh-Bahaei, 2011. Wavelet-based downlink scheduling and resource allocation for long-term evolution cellular systems. IET. Commun., 5: 2091-2095.

Shi, J. and L.L. Yang, 2014. Novel subcarrier-allocation schemes for downlink MC DS-CDMA systems. IEEE. Trans. Wireless Commun., 13: 5716-5728.

Shi, Q., Y.L. Guan, Y. Gong and C.L. Law, 2009. Receiver design for multicarrier CDMA using frequency-domain oversampling. IEEE. Trans. Wireless Commun., 8: 2236-2241.

Simsir, S. and N. Taspinar, 2017. Pilot tones design using grey wolf optimizer for OFDM-IDMA system. Phys. Commun., 25: 259-267.

Yan, X. and G. Liu, 2004. Multiwavelet packet based OFDM system. Proceedings of the 2004 IEEE Asia-Pacific Conference on Circuits and Systems Vol. 2, December 6-9, 2004, IEEE, Tainan, Taiwan, pp: 681-684.

Zakaria, R. and D. Le Ruyet, 2014. Intrinsic interference reduction in a filter bank-based multicarrier using QAM modulation. Phys. Commun., 11: 15-24.

Zarrinkoubm, H., 2014. Understanding LTE with MATLAB: From Mathematical Foundation to Simulation, Performance Evaluation and Implementation. John Wiley \& Sons, Hoboken, New Jersey, USA., ISBN:9781502462565, Pages: 510. 